\title{
Adverse Health Effects of Kratom: An Analysis of Social Media Data
}

\author{
Abdullah Wahbeh \\ Slippery Rock University of PA \\ abdullah.wahbeh@sru.edu
}

\author{
Tareq Nasralah \\ Northeastern University \\ t.nasrala@northeastern.edu
}

\author{
Omar El-Gayar \\ Dakota State University \\ omar.el-gayar@dsu.edu
}

\author{
Mohammad Al-Ramahi \\ Texas A\&M University-San Antonio \\ mohammad.abdel@tamusa.edu
}

\author{
Ahmed El Noshokaty \\ Northern Michigan University \\ aelnosho@nmu.edu
}

\begin{abstract}
This study investigates the adverse healthcare effects associated with the use of kratom. Using machine learning techniques, we analyzed a total of 36,516 users' posts related to kratom. The results and analysis showed that social media could help identify important insights related to the use of kratom. The sentiment and emotion analyses showed that the kratom experience was negative and largely associated with anger, fear, disgust, and sadness. The results from topic modeling showed that kratom is associated with a number of healthcare issues such as rashes and itching, urination, constipation, loss of appetite/weight, dry mouth, seizures, nausea, heartburn, dehydration, hot flashes, and loss of libido. The results indicated that $26 \%$ of users' posts discussed multiple kratom side effects. Also, results showed the prevalence and dominance of loss of libido followed by heartburn, dry mouth, dehydration, and constipation.
\end{abstract}

\section{Introduction}

Kratom tree (Mitragyna speciose) is reportedly found in Southeast Asia as well as parts of Africa [1], with leaves that contain substances that affect a person's mental state. The leaves are traditionally chewed in fresh or dried form to alleviate pain, decrease fatigue, and elevate mood [2, 3]. Recently and due to its potential in alleviating opioid withdrawal symptoms in opioid misuse or abuse such as heroin or morphine [4, 5], kratom use has spread to Europe and the United States (US), where abuse potential and health hazards emerged increasingly [6].

Recently, kratom use in the US has increased significantly with the belief that kratom is less harmful than opioid drugs. Kratom is used in the US as a dietary supplement and for its stimulant effects and as an opioid substitute. Kratom related exposure brought the attention of the public to kratom use where the total kratom-related calls received by poison control centers were significantly increase [7]. An estimate of $7.4 \%$ of kratom reported calls were classified as major, life threatening with some residual disability [2]. More recently, epidemiologic and laboratory evidence indicated that kratom was the likely source of a multistate outbreak of Salmonella infections, where Seventy-six (74\%) of 103 people interviewed reported consuming kratom in pills, powder, or tea [8].

With the rising concerns regarding kratom safety [9], several studies reported negative side-effects associated with kratom use (e.g., [6, 10-12]). However, we are not aware of any study that attempts to examine large-scale social media data to understand users' health reported issues. Social media is considered a promising and viable source of data for gaining insights into kratom's health effects [13] that can enrich existing literature [12].

Accordingly, the objective of this research is to investigate kratom adverse health issues by collecting and analyzing recent social media posts. Specifically, we collected posts related to experiences with kratom use from Reddit and Twitter. Using unsupervised machine learning technique, topic mining, we analyzed the data to understand the recent themes and perceptions about the adverse health effects of kratom. In addition, we used a supervised machine learning technique, the ReadMe algorithm, to analyze the relative prevalence of the adverse effects discovered in users' posts.

This study informs existing literature regarding the adverse health effects of kratom and the relative prevalence of these effects in social media discussions. Furthermore, from a methodological perspective, this research, as opposed to existing literature, adopts a big dataset collected from social media and leverages data analytics techniques to analyze data. From a practical perspective, findings highlight important concerns and healthcare related issues of kratom use, which could help inform regulations, research, interventions, and awareness aimed at mitigating the adverse effects from kratom use. 
The remainder of the paper is organized as follows: the next section provides an overview of existing literature related to the health impact of kratom. The research design and methodology section presents the data collection, preparation, and analysis. The results section highlights the topics identified, and the discussion section depicts the key findings from the study. The paper concludes with a summary of contributions and limitations.

\section{Literature review}

\subsection{Adverse drug events on social media}

Every year Adverse Drug Events (ADEs) are known to be responsible for 98,000 deaths in the USA [14]. Classical methods of identifying the ADEs from studying report statements, expert knowledge, and staff operated record reviews are perceived as underrated in terms of reporting ADEs or ADRs Adverse Drug Reactions [15]. Theses traditional reporting channels might not be sufficient given the massive toll of ADEs on people's lives.

Social media analytics could describe ADEs adverse drug events in real-time before official reporting. Studying ADEs from publicly available drug users' data through social media analytics is not new. Text mining methodologies were proposed to detect ADEs from publicly available twitter data through supervised learning [16] or semi-supervised learning [17]. The ADEs of specific drugs like Baclofen, Duloxetine, Gabapentin, Glatiramer were extracted through supervised learning models giving a deeper understanding of ADEs from spontaneous tweets of drug users[18]. Also, other studies of ADEs studied the ADEs and ADRs of drugs related to specific chronic diseases like diabetes and heart diseases $[19,20]$.

\subsection{Adverse effect of Kratom}

Following methodologies such as surveys [2, 16], systematic reviews [17, 18], field studies [10], and qualitative methods [11], several studies have addressed the general adverse effects associated with kratom use $[2,16,17,20,21]$ as well as kratom effect on mental health [12] and cognitive functions [10].

Swogger et al. [11] have analyzed users' selfreported experience with Kratom using conventional content analysis. Following a coding procedure, participants reported negative experiences with kratom related to nausea and stomachache $(16.1 \%)$, sweats $(9.3 \%)$, dizziness $(6.8 \%)$, vomiting $(5.6 \%)$, and itching $(3.1 \%)$. Other experiences are related to mouth numbness, visual alterations, and sedation.
Grundmann [2] analyzed survey data to address the benefits and side effects reported by kratom users. Using binomial logistic regression, results showed that negative experience with kratom includes nausea $(12.75 \%)$, constipation $(9.17 \%)$, and dizziness/ drowsiness $(4.81 \%)$, diarrhea $(0.49 \%)$, vomiting (4.01\%), heart palpitations $(0.71 \%)$, shortness of breath $(0.22 \%)$, stomach upset $(4.7 \%)$, fainting $(0.14 \%)$, irritability or agitation $(2.4 \%)$, and high blood pressure (hypertension) $(0.28 \%)$.

Systematic literature reviews have addressed kratom abuse [21], kratom use, and mental health [12], adverse effects of kratom [21], and differences between traditional and non-traditional kratom users [22]. The reviews showed that kratom has some opioid effects, but low respiratory depression and abuse potential [21], high dosage kratom has anxiolytic effects [12], kratom can cause loss of weight, dehydration, constipation, fatigue, shaking of hands, headaches, tiredness, loss of appetite, stomachaches, alternating between chills and sweats, dizziness, itching, numbness in the mouth and throat, sedation, visual alterations, unsteadiness [22], tachycardia $(25 \%)$, hypertension $(12 \%)$, agitation or irritability $(24 \%)$, drowsiness $(19 \%)$, nausea $(15 \%)$, confusion, tremor, diaphoresis, hypothyroidism, intrahepatic cholestasis [23], nausea, stomachache, and cramping (16.1\%), alternating chills and sweats (9.3\%), dizziness and unsteadiness (6.8\%), vomiting (3.1\%), neutral experiences $(10 \%)$, which included numbness of the throat and mouth, visual alterations, and sedation [21], agitation (18.6\%), tachycardia (16.9\%), drowsiness (13.6\%), vomiting (11.2\%), and confusion $(8.1 \%)$, serious effects of seizure $(6.1 \%)$, withdrawal $(6.1 \%)$, hallucinations $(4.8 \%)$, respiratory depression $(2.8 \%)$, coma $(2.3 \%)$, and cardiac or respiratory arrest $(0.6 \%)$ [24].

According to literature and up to the knowledge of the researchers, no study has been conducted to identify the adverse healthcare effects associated with kratom and their predominance from large datasets such as that obtained from social media content using social media analytics and data mining techniques.

\section{Research design and methodology}

To investigate kratom use health related issues, we collected and analyzed social media related posts. We collected the study dataset using Crimson Hexagon (now Brandwatch), a social media data collection and analytics tool [25]. For data analysis, we applied sentiment, topic modeling, and supervised learning analysis on the collected dataset. 


\subsection{Data collection and pre-processing}

The data was collected from Reddit, and Twitter social media using Crimson Hexagon and employing the query shown in Figure 1. We collected all posts from 01-01-2015 to 05-22-2020. To retrieve the relevant posts, we used the search query in figure 1, that includes several health-related keywords [26]. The collected posts are all selected based on the criteria of having at least one health related keyword and kratom related keyword. The size of data collected is 36,516 posts, with $96.8 \%$ collected from Reddit.

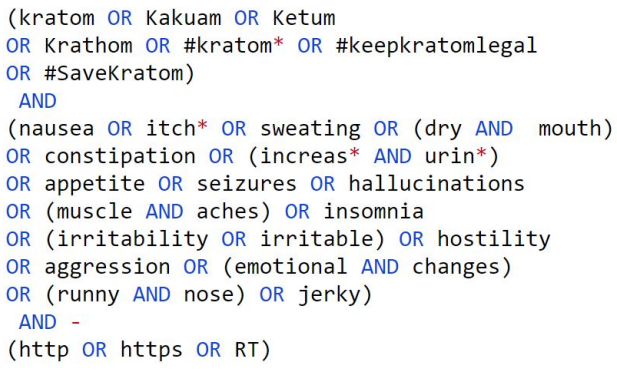

Figure 1: Search Query

To prepare the posts for topic modeling, we removed numbers, URLs, stop words, and represented each post using the well-known Term Frequency Inverse Document Frequency (TF-IDF) weighting scheme $[27,28]$. Particularly, the weight of a word $i$ in a user post $j$ is given by:

$$
F_{i, j} * \log (N / D F)
$$

Where $F_{i, j}$ is the frequency of the word $i$ in post $j$, $N$ indicates the number of posts in the data set, and $D F$ is the number of discussion posts that contain word $i$.

\subsection{Topic Modeling}

Topic models are types of statistical algorithms for extracting the main topics in a collection of documents. Latent Dirichlet Allocation (LDA) is one of the common topic modeling algorithms used [29]. The algorithm produces a set of topics with a probability distribution over words in each topic.

The algorithm also generates probability distributions over topics for each document $[30,31]$. To explain the outputs of LDA, assume $D=\left\{d_{1}, d_{2}\right.$, $\ldots, d_{\mathrm{n}\}}$, the set of documents in a collection, $T=\left\{t_{1}, t_{2}\right.$, $\ldots, t_{\mathrm{m}\}}$, the set of generated topics, and $\mathrm{W}=\left\{w_{1}, w_{2}\right.$, $\ldots, w_{\mathrm{k}\}}$ the words in each topic. The first output is a $D$ $\times T$ matrix with $n \times m$ size, where the weight $w_{\mathrm{i}, \mathrm{j}}$ is the association between a document $d_{\mathrm{i}}$ and a topic $t_{\mathrm{j}}$. In our study, the documents are discussion posts for users. The second result is a $T \times W$ matrix with $m \times k$ size, where the weight $w_{\mathrm{i}, \mathrm{j}}$ is the association between the topic $t_{\mathrm{i}}$ and a word $w_{\mathrm{j}}$. The corresponding reproductive process is shown below [30, 32]:

(1) For each topic $t \in\{1, \ldots, m\}$,

(a) generate a probability distribution over words $\beta t \sim \operatorname{Dirichlet}(\eta)$.

(2) For each document $d$,

(a) generate a vector of the topic probability distribution

$$
\theta d \sim \operatorname{Dirichlet}(\alpha) .
$$

(b) For each word $w_{\mathrm{i}}$ in document $d$,

(i) generate a topic assignment $z_{\mathrm{i}} \sim \operatorname{Multinomial}(\theta d)$

(ii) generate a word $w_{n} \sim \operatorname{Multinomial}\left(\beta_{z i}\right)$

$\beta t$ is the word distribution for topic $t$, and $\theta d$ is the topic distribution for document $d$. The notations $\eta$ and $\alpha$ are model parameters.

We used Python to build and run LDA algorithm and identified 50 topics and within each topic showed the top-10 words and their relative weight (i.e. probability). The naming of topics was first conducted by the first author and confirmed by the second author. Naming was initially based on the identification of a logical connection between the ten most frequent words for a topic.

\subsection{Supervised Learning}

Crimson Hexagon employs the ReadMe algorithm developed by Daniel Hopkins and Gary King [33]. This is a supervised learning algorithm that expects the researcher to hand-code a "training set" of documents (posts) into a set of predefined categories. Crimson Hexagon provides an already 'trained' model for sentiment and opinion mining, or an opportunity for the researcher to train their own model using userdefined categories.

The ReadMe algorithm is particularly suited when the objective is to know the proportion of the population of posts that fit in specific categories. Rather than calculating these proportions based on the categorization of individual posts, ReadMe gives approximately unbiased estimates of category proportions even when the optimal classifier performs poorly [33].

In this research, we used the ReadMe algorithm to analyze the proportion of tweets and Reddit posts that fall into specific categories. We initially utilized the topics derived from LDA algorithm to be the initial categories. In addition, to account for lengthy posts that discuss multiple kratom adverse side effects, we have defined a new category "More than one" to represents such posts and avoid any bias when training and running the ReadMe algorithm. We then initially trained the algorithm on 300 tweets and Reddit posts 
following the codebook in appendix A, and then ran the algorithm iteratively on the remaining posts. The training was done iteratively, ensuring that the examples clearly outline each category.

\section{Results}

\subsection{Sentiment analysis}

We conducted sentiment and emotion analysis on the collected posts. Sentiment analysis is an emerging area of Natural Language Processing (NLP) used to determine whether the text contains a positive or a negative polarity [34]. In addition, emotion analysis provides an additional layer of contextual analysis, utilizing the "Ekman 6" (Anger, Fear, Disgust, Joy, Surprise, and Sadness) basic human emotions [35].

Figure 2 summarizes the results of both analyses for the collected posts. The emotion analysis results show that $34.3 \%$, about one-third, of the posts were reflecting anger emotion, which indicates users' outrage against kratom in general. Example posts reflecting the online users' anger include, "If you're getting seizures from kratom, you have to stop. It isn't worth it, man, regardless if you're worthless." Sentiment Analysis (Positive/Negative) results show that $87.3 \%$ of the posts were classified as a negative sentiment.

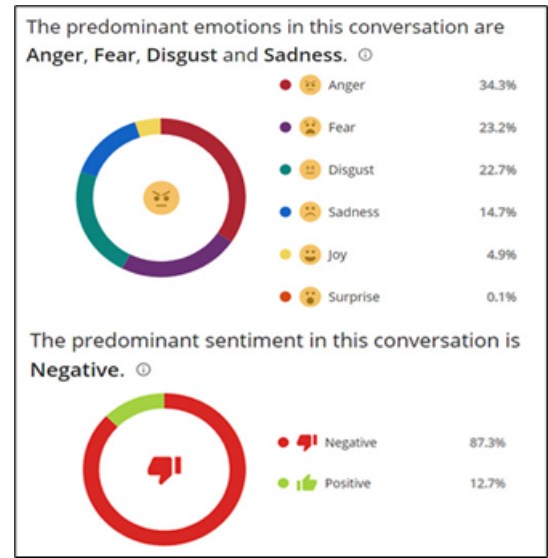

Figure 2: Emotion and Sentiment Analysis

\subsection{Trend analysis}

The distribution of kratom posts over the identified topics from the supervised learning is shown in Figure 3. Overall, results show that posts that discussed more than one kratom adverse side effect account for $26 \%$ of the total posts followed by loss of libido $(16 \%)$ and heartburn $(10 \%)$. Each of the remaining adverse side effects was discussed in less than $10 \%$ of the total kratom related posts.

As shown in Figure 3, the volume of posts over time by category. The number of posts has increased over time, especially starting Mach 2017. Furthermore, there was an increase in posts that discuss multiple kratom adverse effect as well as posts that focused on the loss of libido, heartburn, and recently dehydration.

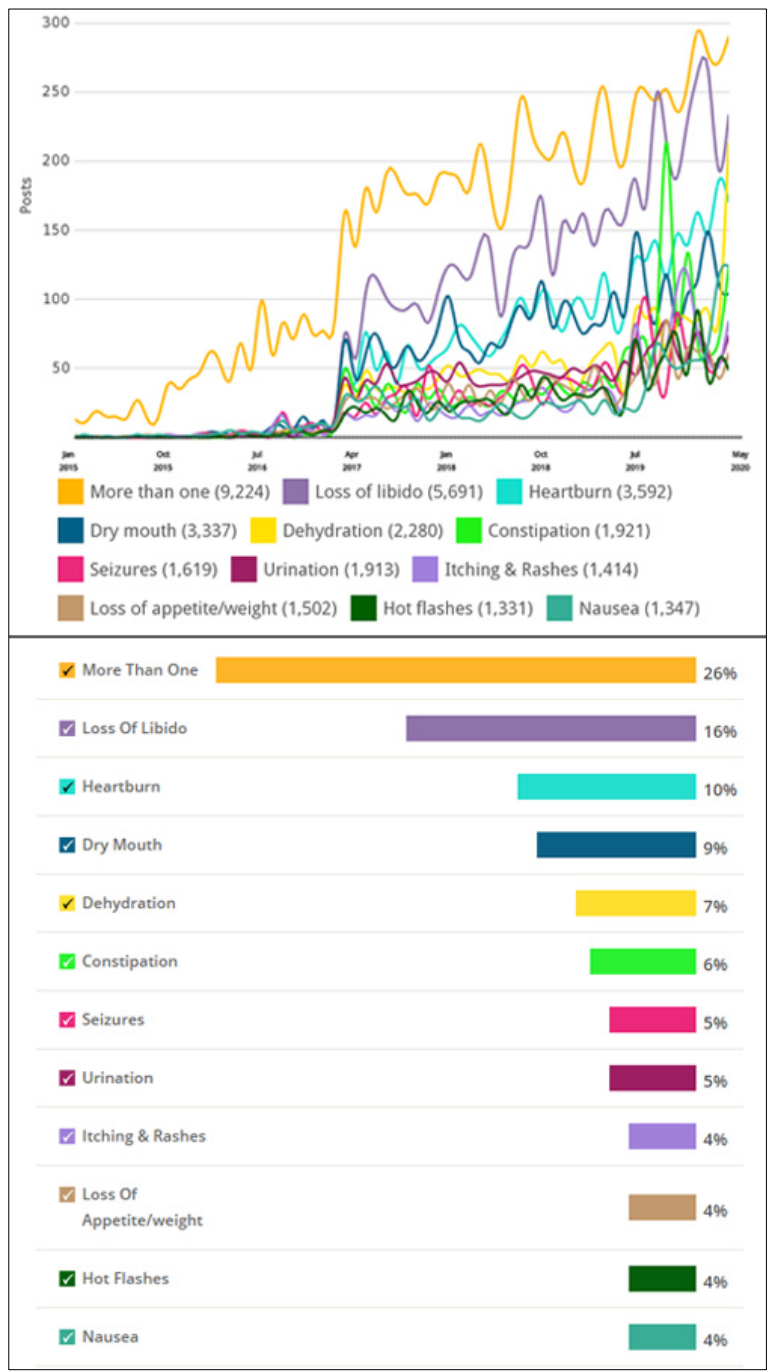

Figure 3: Percentages of Posts/ Categories

\subsection{Topics analysis}

We examined the LDA model results and manually labeled and grouped 12 health issues topics from the 50 topics of the model results. These issues were mainly related to rashes and itching, urination, constipation, loss of appetite/weight, dry mouth, 
seizures, nausea, heartburn, dehydration, hot flashes, and loss of libido.

The use of kratom could cause rashes and itching. According to Figure 4, posts related to "itchy," "body," "skin," "rashes," and "red" dominated this category. Example posts include "Hey I get rashes too! My skin is a mess from Kratom." , "Every time I took kratom, I got rash", and "I've noticed that when I take green kratom I become a hot, itchy and grouchy person."

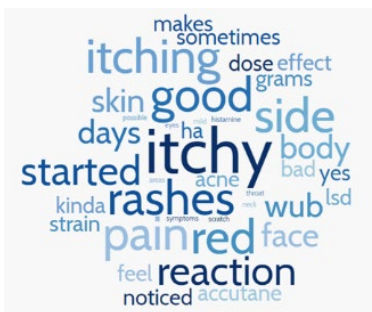

Figure 4. Rashes and Itching

The use of kratom could cause urination. According to Figure 5, posts related to "urination", "urinary", "urinate", "cause", and "increased" dominated this category. Example posts include "I've experienced urinary retention after my kratom dose has kicked in. There's several anecdotes of various urinary problems caused by Kratom on this sub and elsewhere." and "Increased urination is the single most annoying thing about kratom for me and also the only negative."

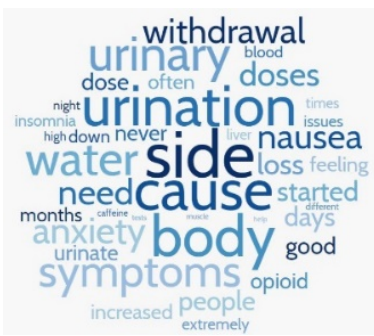

Figure 5. Urination

The use of kratom could cause constipation. According to Figure 6, posts related to "constipation", "effects", "drink", "magnesium", and "cause" dominated this category. Example posts include "I like kratom but due 2 the severe constipation l only use a small ant bt still constipation persts." and "The only bad experience I've had with kratom is constipation but I like to switch my stimulants so I don't get a tolerance so much."

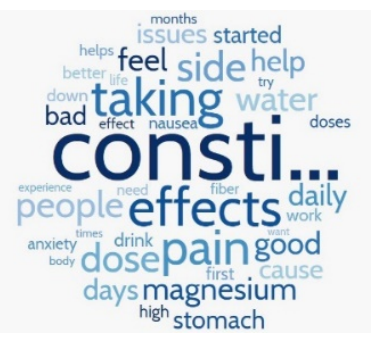

Figure 6. Constipation

The use of kratom could cause loss of appetite/weight. According to Figure 7, posts related to "loss", "appetite", "weight", "eat", and "effects" dominated this category. Example posts include, "I' $m$ a 33 year old female and it caused me to lose about 10 pounds...even though I'm tapering off I'm still losing. I don't have an appetite until 7-8 at night" and " when I use kratom my appetite goes away at least for a while."

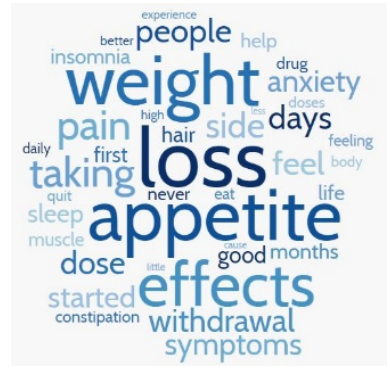

Figure 7. Loss of Appetite/Weight

The use of kratom could cause dry mouth. According to Figure 8, posts related to "dry", "mouth", "water", "drink", and "tongue" dominated this category. Example posts include "For whatever reason I find when I'm taking kratom every day for a few days my mouth is constantly dry and I'm Always thirsty even if I drink tons of water." and "I don't toss $n$ wash but I drink a lot of water anyway but started drinking even more because of dry mouth after starting kratom".

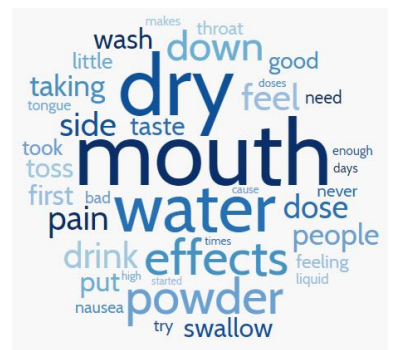

Figure 8. Dry Mouth

The use of kratom could cause seizures. According to Figure 9, posts related to "seizure," "tonic," "clonic," "cause," and "disorder" dominated this category. Example posts include "A couple 
months ago in front of my wife, eating pizza, and on a whopping dose of kratom (for my tolerance) I had a full tonic clonic seizure." and "I told them what I took. Thankfully my neurologist has experience with people having seizures after taking kratom".

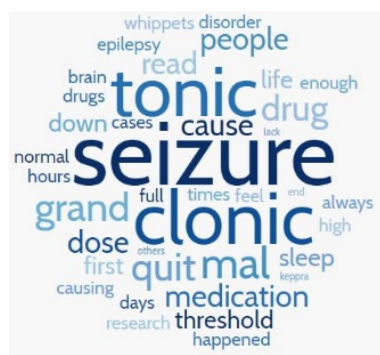

Figure 9. Seizures

The use of kratom could cause nausea. According to Figure 10, posts related to "nausea," "stomach," "doses," and "experience" dominated this category. Example posts include "I always had issues with nausea with kratom. Is that just me? Or does it depend on the dose?", "kratom is so hit/miss/everything in between for me. Sometimes I get a nice buzz, sometimes I get nausea, sometimes I feel nothing" and "I've experienced kratom nausea twice, and it was two times too many. Not even worth building tolerance to in my book.".

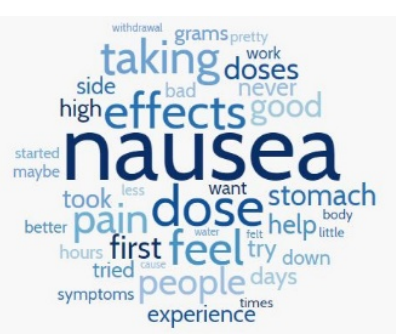

Figure 10. Nausea

The use of kratom could cause heartburn. According to Figure 11, posts related to "heartburn," "stomach," "acid," and "experience" dominated this category. Example post include "Newby to kratom. First month was great. I had no issues (started with capsules and then TW with various strains). Last week I started getting what seemed like heartburn".

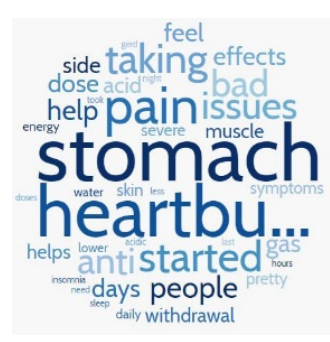

Figure 11. Heartburn
The use of kratom could cause dehydration. According to Figure 12, posts related to "dehydration," "water," "drink," and "cause" dominated this category. Example posts include "I'm afraid that with kratom the dehydration could become a medical issue" and "kratom took away my appetite to the point of malnutrition and dehydration".

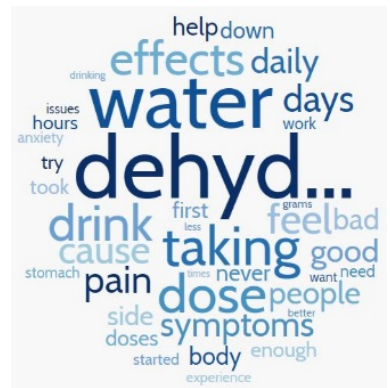

Figure 12. Dehydration

The use of kratom could cause hot flashes. According to Figure 13, posts related to "hot" and "flashes" dominated this category. Example posts include "RLS, insomnia, cold sweats, hot flashes, anxiety, depression, moodiness, awful runny shits are all things I experience when quitting kratom." and "I've been taking kratom for about 2 months now, but never while with a cold. My symptoms are hot flashes, headache, sore throat, runny nose".

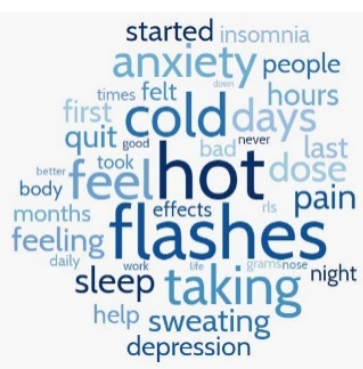

Figure 13. Hot Flashes

The use of kratom could cause loss of libido. According to Figure 14, posts related to "loss," "libido," and "sex" dominated this category. Example post includes "I personally notice a reduced libido on the days I take kratom.". 


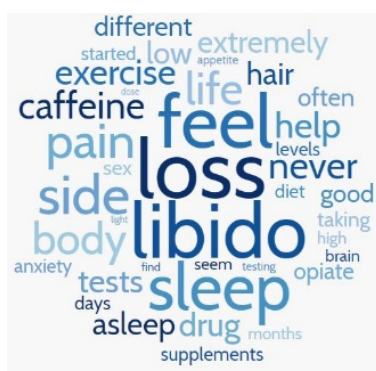

Figure 14. Loss of Libido

\section{Discussion}

Data collection and analysis showed that social media could help identify important insights related to adverse drug events, specifically healthcare adverse effects associated with the use of kratom. The period from 01-01-2015 to 05-22-2020 saw a dramatic increase in the discussions about kratom on social media, specifically, Reddit. Such a dramatic increase could be used as a gauge and indicator to reflect the increase in kratom use in the US. Furthermore, as shown in trend analysis, discussions about kratom adverse side effects also increased significantly.

The sentiment and emotion analysis results showed that the negative kratom experience, according to the collected posts and discussions, was largely associated with anger, fear, disgust, and sadness. This indicates that while some people are using kratom with the belief that it is not as harmful as other substances, the evidence from social media indicates that kratom use can cause adverse healthcare effects, and the fact that kratom is much riskier than the majority of kratom users tend to believe.

Kratom is associated with a number of adverse healthcare related issues. The reported adverse healthcare effects of kratom appear to vary from one person to another and depends on a number of factors such as the dose, existing condition, and the level of addiction - regular and non-regular users. As reported by a number of posts, the body could have an inflammatory response to kratom, which could lead to rashes and itching. In addition, the frequent use of kratom could cause problems related to the digestive system in terms of constipation. Despite the fact that kratom is not a weight-loss supplement, its functioning can cause weight loss and loss of appetite for regular users [36].

Kratom has diuretic effects [36], which cause water to be released from the body. Such effects could lead to frequent urination, dry mouth, and dehydration. Kratom products are being adulterated with psychoactive substances [37], which could lead to seizures. Furthermore, kratom ingestion could cause hangover-like symptoms [21] such as nausea, which can provoke puking and vomiting. In addition, kratom ingestion can result in acid reflux, which in turn causes heartburn. Finally, kratom could cause loss of libido and hot flashes, the sudden feeling of heat in the chest, face, and head.

The majority of the collected posts and discussions about kratom discussed more than one side effect. This could be used as clear evidence that kratom could cause multiple complications at the same time. Furthermore, posts about the loss of libido and heartburn were also significant. These are interesting findings as compared to the surveyed literature where studies have not reported the loss of libido and heartburn as a frequent side effect from kratom. These side effects were reported by many online users where many stated that "kratom has destroyed my testosterone and libido," "taking kratom every day lowers testosterone and libido," "I use Kratom every day, so my sex drive is lower," "the kratom is killing me and I have heartburn," "my dad did a bunch of kratom yesterday to the point that it made him sick with really bad heartburn," and "kratom and psoriasis has now given me heartburn." Finally, other side effects such as loss of weight and appetite, itching [11, 22], dehydration [22], seizure [24], nausea [2, 11, 21, $23]$, constipation $[2,22]$ are pretty consistent with existing literature. Other reported side effects such as dry mouth and urination could be related to dehydration.

The study demonstrated the potential of social media analytics for reporting adverse drug effects, as in the case of kratom. With the powerful combination of social media data and cutting-edge analytics, as well as the need to increase the awareness regarding kratom use side effects, we can see that change is on the horizon. Using social media analytics can help identify the adverse effect associated with the use of kratom in real time, which in turn could influence and improve decision making and safety with respect to kratom legalization, use, and dose. Also, it can help increase public awareness by highlighting kratom adverse healthcare effects from the public perspective and from kratom's current users.

Moreover, the methodology adopted in this study provides a real-time surveillance approach to monitor adverse drug effects reported in social media posts and to analyze user sentiment and emotion. This approach, in turn, could help alleviate the shortcoming and limitations associated with traditional approaches for identifying and reporting adverse drug effects as well as supplement existing approaches for reporting adverse drug effects [38]. 


\section{Conclusion and Limitations}

In this research, we explored the adverse healthcare effects related to kratom use by collecting and analyzing recent social media posts from Reddit and Twitter. We used machine learning techniques, topic modeling, to automatically analyze the content of the collected posts. Results showed several healthrelated issues are being discussed on social media. These issues are mainly related to itches, urination, constipation, Loss of appetite/weight, Rashes, Dry mouth, seizures, Nausea, heartburn, Dehydration, Hot flashes, and Loss of libido.

This research expanded the existing literature on the use of social media data to address the health impacts of kratom. Our findings support existing literature that reported nausea [2, 11, 22], itching, loss of weight, dehydration [11], seizures [23, 39], and constipation $[11,22]$. Furthermore, we were able to identify more issues related to urination, dry mouth, heartburn, hot flashes, and loss of libido.

This research is not without any limitation. First, a challenging part of data analysis using topic modeling is handling short, focused posts from Twitter with lengthy, detailed posts from Reddit. Furthermore, the reported results are based on the general discussion about kratom on social media. It is more informative to report the adverse healthcare effects of kratom based on self-identified kratom addicted users. As future research, we plan to investigate the conditions and factors that could lead to specific kratom adverse side effects, such as the dose, existing condition, use of other drugs, and the level of addiction.

\section{References}

[1] L. C. Thuan, "Addiction to Mitragyna speciosa," Proceedings of the Alumni Association, vol. 10, pp. 322-324, 19571957.

[2] O. Grundmann, "Patterns of kratom use and health impact in the US-results from an online survey," Drug and alcohol dependence, vol. 176, pp. 63-70, 2017.

[3] M. L. Warner, N. C. Kaufman, and O. Grundmann, "The pharmacology and toxicology of kratom: from traditional herb to drug of abuse," International journal of legal medicine, vol. 130, no. 1, pp. 127-138, 2016.

[4] E. W. Boyer, K. M. Babu, J. E. Adkins, C. R. McCurdy, and J. H. Halpern, "Self-treatment of opioid withdrawal using kratom (Mitragynia speciosa korth)," (in en), Addiction, vol. 103, no. 6, pp. 1048-1050, 20082008.

[5] Z. Hassan et al., "From Kratom to mitragynine and its derivatives: Physiological and behavioural effects related to use, abuse, and addiction," (in en), Neuroscience \& Biobehavioral Reviews, vol. 37, no. 2, pp. 138151, February 1, 20132013.

[6] D. Singh, C. P. Müller, and B. K. Vicknasingam, "Kratom (Mitragyna speciosa) dependence, withdrawal symptoms and craving in regular users," Drug and alcohol dependence, vol. 139, pp. 132-137, 2014.

[7] M. Anwar, R. Law, and J. Schier, "Notes from the Field : Kratom ( Mitragyna speciosa) Exposures Reported to Poison Centers - United States, 2010-2015," (in en), MMWR. Morbidity and Mortality Weekly Report, vol. 65, no. 29, pp. 748-749, 2016-07-29 2016.

[8] CDC. (2019). Multistate Outbreak of Salmonella I 4,[5],12:b:- Infections Linked to Kratom Products $\mid$ February $2018 \mid$ Salmonella $\mid$ CDC.

[9] C. Veltri and O. Grundmann, "Current perspectives on the impact of Kratom use," (in en), Substance Abuse and Rehabilitation, vol. Volume 10, pp. 23-31, 07/2019 2019.

[10] D. Singh et al., "Long-term cognitive effects of Kratom (Mitragyna speciosa Korth.) use," Journal of psychoactive drugs, vol. 51, no. 1, pp. 19-27, 2019.

[11] M. T. Swogger et al., "Experiences of kratom users: a qualitative analysis," Journal of psychoactive Drugs, vol. 47 , no. 5 , pp. 360-367, 2015.

[12] M. T. Swogger and Z. Walsh, "Kratom use and mental health: A systematic review," Drug and Alcohol Dependence, vol. 183, pp. 134-140, 2018.

[13] T. Nasralah, O. El-Gayar, and Y. Wang, "What Social Media Can Tell Us About Opioid Addicts: Twitter Data Case Analysis," AMCIS 2019 Proceedings, 2019-07-12 2019.

[14] C. Emmanuel, P. Cristian, M. Beatrice, F. Gregoire, and B. Regis, "Data-Mining-Based Detection of Adverse Drug Events," (in en), Studies in Health Technology and Informatics, pp. 552-556, 20092009.

[15] J. Lardon et al., "Adverse Drug Reaction Identification and Extraction in Social Media: A Scoping Review," Journal of Medical Internet Research, vol. 17, no. 7, 2015-7-10 2015.

[16] S. Katragadda, H. Karnati, M. Pusala, V. Raghavan, and R. Benton, "Detecting adverse drug effects using link classification on twitter data," in 2015 IEEE International Conference on Bioinformatics and Biomedicine (BIBM), 2015, pp. 675-679.

[17] K. Lee et al., "Adverse Drug Event Detection in Tweets with Semi-Supervised Convolutional 
Neural Networks," 2017, pp. 705-714, Perth, Australia: International World Wide Web Conferences Steering Committee.

[18] Y. Peng, M. Moh, and T.-S. Moh, "Efficient adverse drug event extraction using Twitter sentiment analysis," in 2016 IEEE/ACM International Conference on Advances in Social Networks Analysis and Mining (ASONAM), 2016, pp. 1011-1018.

[19] X. Liu and H. Chen, "Identifying adverse drug events from patient social media: A case study for diabetes," (in en), IEEE Intelligent Systems, vol. 30, no. 3, pp. 44-51, 5/2015 2015.

[20] X. Liu, J. Liu, and H. Chen, "Identifying Adverse Drug Events from Health Social Media: A Case Study on Heart Disease Discussion Forums," in International Conference on Smart Health, 2014.

[21] J. E. Henningfield, R. V. Fant, and D. W. Wang, "The abuse potential of kratom according the 8 factors of the controlled substances act: implications for regulation and research," Psychopharmacology, vol. 235, no. 2, pp. 573589, 2018.

[22] D. Singh, S. Narayanan, and B. Vicknasingam, "Traditional and non-traditional uses of Mitragynine (Kratom): a survey of the literature," Brain Research Bulletin, vol. 126, pp. 41-46, 2016.

[23] C. Michael White, "Pharmacologic and clinical assessment of kratom," The Bulletin of the American Society of Hospital Pharmacists, vol. 75, no. 5, pp. 261-267, 2018.

[24] W. Eggleston, R. Stoppacher, K. Suen, J. M. Marraffa, and L. S. Nelson, "Kratom Use and Toxicities in the United States," (in en), Pharmacotherapy: The Journal of Human Pharmacology and Drug Therapy, vol. 39, no. 7, pp. 775-777, 20192019.

[25] Brandwatch. (2020). Crimson Hexagon and Brandwatch. Available: https://www.brandwatch.com/p/crimsonhexagon/

[26] N. I. o. D. NIDA. (2019). Kratom DrugFacts. Available: https://www.drugabuse.gov/publications/drugfa cts/kra

[27] E. Haddi, X. Liu, and Y. Shi, "The Role of Text Pre-processing in Sentiment Analysis," (in en), Procedia Computer Science, vol. 17, pp. 26-32, 20132013.

[28] J. Ramos, "Using TF-IDF to Determine Word Relevance in Document Queries," 2003, pp. 133142, NJ: Proceedings of the first instructional conference on machine learning: Piscataway.
[29] D. M. Blei, A. Y. Ng, and M. I. Jordan, "Latent dirichlet allocation," Journal of machine Learning research, vol. 3, no. Jan, pp. 993-1022, 20032003.

[30] M. A. Al-Ramahi, J. Liu, and O. F. El-Gayar, "Discovering design principles for health behavioral change support systems: A text mining approach," ACM Transactions on Management Information Systems (TMIS), vol. 8, no. 2-3, pp. 1-24, 20172017.

[31] Y. Bao and A. Datta, "Simultaneously discovering and quantifying risk types from textual risk disclosures," Management Science, vol. 60, no. 6, pp. 1371-1391, 20142014.

[32] M. Al-Ramahi and C. B. Noteboom, "Mining User-Generated Content of Mobile Patient Portal: Dimensions of User Experience," $A C M$ Transactions on Social Computing, vol. 3, no. 3, pp. 1-24, 20202020.

[33] D. J. Hopkins and G. King, "A Method of Automated Nonparametric Content Analysis for Social Science," (in en), American Journal of Political Science, vol. 54, no. 1, pp. 229-247, 20102010.

[34] S. Mansour, "Social Media Analysis of User's Responses to Terrorism Using Sentiment Analysis and Text Mining," (in en), Procedia Computer Science, vol. 140, pp. 95-103, 2018 2018.

[35] P. Ekman, "Facial expression and emotion," American psychologist, vol. 48, no. 4, p. 384, 1993.

[36] D. Singh et al., "Evaluating the hematological and clinical-chemistry parameters of kratom (Mitragyna speciosa) users in Malaysia," (in en), Journal of Ethnopharmacology, vol. 214, pp. 197-206, 03/2018 2018.

[37] D. Fluyau and N. Revadigar, "Biochemical Benefits, Diagnosis, and Clinical Risks Evaluation of Kratom," (in English), Frontiers in Psychiatry, vol. 8, 20172017.

[38] D. Pappa and L. K. Stergioulas, "Harnessing social media data for pharmacovigilance: a review of current state of the art, challenges and future directions," (in en), International Journal of Data Science and Analytics, vol. 8, no. 2, pp. 113-135, 2019-09-01 2019.

[39] B. Vicknasingam et al., "Kratom and Pain Tolerance: A Randomized, Placebo-Controlled, Double-Blind Study," The Yale Journal of Biology and Medicine, vol. 93, no. 2, pp. 229238, 2020-6-29 2020. 


\section{Appendix A: Codebook for Labeling Categories}

\begin{tabular}{|c|c|c|c|}
\hline & Category & Keywords & Examples \\
\hline 1 & $\begin{array}{l}\text { Itching \& } \\
\text { Rashes }\end{array}$ & $\begin{array}{l}\text { itchy, body, } \\
\text { antihistamine, } \\
\text { Benadryl...etc. }\end{array}$ & Itching face from kratom \\
\hline 2 & Urination & $\begin{array}{l}\text { urination, urinate, } \\
\text { urinary, } \\
\text { urine...etc. }\end{array}$ & $\begin{array}{l}\text { Kratom has coffee like effects. Makes you urinate blah blah. Lyrica } \\
\text { has been associated with nephrotoxicity. I would just say drink a shit } \\
\text { ton of water. Not quite until your urine is clear (that means you've } \\
\text { flushed your electrolytes) but just until it has that yellow tinge. }\end{array}$ \\
\hline 3 & $\begin{array}{l}\text { Loss of } \\
\text { appetite }\end{array}$ & $\begin{array}{l}\text { appetite, weight, } \\
\text { eat...etc. }\end{array}$ & $\begin{array}{l}\text { Kratom has always given me unhealthy (sickly) weight loss. It does } \\
\text { kill my appetite, but I still feel like it isn't enough to explain the drop. }\end{array}$ \\
\hline 4 & Constipation & $\begin{array}{l}\text { constipation, drink, } \\
\text { magnesium...etc. }\end{array}$ & $\begin{array}{l}\text { I was constantly constipated, sometimes being backed up for a week } \\
\text { (don't know how it's even possible). I have stopped taking oxy a } \\
\text { year ago and now take kratom. }\end{array}$ \\
\hline 5 & Dry mouth & $\begin{array}{l}\text { mouth, dry, } \\
\text { powder, taste...etc. }\end{array}$ & $\begin{array}{l}\text { I can't stand toss and wash, the dry powder hitting my mouth is so } \\
\text { gross, not to mention the times I sucked it up into my sinuses }\end{array}$ \\
\hline 6 & Nausea & $\begin{array}{l}\text { nausea, stomach, } \\
\text { doses, } \\
\text { experience....etc. }\end{array}$ & $\begin{array}{l}\text { Kratom itself is a joke. Huge waste of time and money, all I got was } \\
\text { a few hours worth of nausea. }\end{array}$ \\
\hline 7 & Seizures & $\begin{array}{l}\text { seizures, tonic, } \\
\text { clonic, cause, } \\
\text { disorder...etc. }\end{array}$ & $\begin{array}{l}\text { Kratom is obviously a very dangerous substance. Excellent seizures } \\
\text { by you and the Border Force. }\end{array}$ \\
\hline 8 & Heartburn & $\begin{array}{l}\text { heartburn, } \\
\text { stomach, acid, } \\
\text { experience...etc. }\end{array}$ & $\begin{array}{l}\text { Kratom and Beer! I get this effect often although sometimes I enjoy a } \\
\text { beer or 2, anymore than that and the heartburn gets pretty bad. }\end{array}$ \\
\hline 9 & Dehydration & $\begin{array}{l}\text { Dehydration, } \\
\text { water, drink, } \\
\text { cause...etc. }\end{array}$ & $\begin{array}{l}\text { I got so dehydrated I really thought I was dying, and I've never had } \\
\text { that feeling before. It was HORRIBLE. Worse than any opiate or } \\
\text { benzo withdrawal }\end{array}$ \\
\hline 10 & Loss of libido & Libido, sex...etc. & $\begin{array}{l}\text { I wasted a lot of money and time using it. It killed my sex drive and } \\
\text { left me feeling empty and lost. }\end{array}$ \\
\hline 11 & Hot flashes & $\begin{array}{l}\text { RLS, insomnia, } \\
\text { cold sweats, hot } \\
\text { flashes, } \\
\text { depression...etc. }\end{array}$ & $\begin{array}{l}\text { My Kratom Addiction, If I Can Help ONE Person Get Off That Crap } \\
\text { Then This Insomnia-Induced Post About My Struggle With It Is So } \\
\text { Worth It }\end{array}$ \\
\hline 12 & $\begin{array}{l}\text { More than } \\
\text { one }\end{array}$ & & $\begin{array}{l}\text { Using the Kratom shots now since I was taking like } 30 \mathrm{~g} \text { of powder a } \\
\text { few times a day and choking it down was causing me to almost vomit } \\
\text { every time. Also, I can't stop shitting, sweating, or drinking water. I } \\
\text { can feel the lethargy starting to set in and the RLS at my desk. }\end{array}$ \\
\hline
\end{tabular}

